\title{
Three-Component Fermi Gas in a one-dimensional Optical Lattice
}

\author{
P. Azaria, ${ }^{1}$ S. Capponi,${ }^{2,3}$ and P. Lecheminant ${ }^{4}$ \\ ${ }^{1}$ LPTMC, Université Pierre et Marie Curie, CNRS, 75005 Paris, France. \\ ${ }^{2}$ Université de Toulouse; UPS; Laboratoire de Physique Théorique (IRSAMC); F-31062 Toulouse, France \\ ${ }^{3}$ CNRS; LPT (IRSAMC); F-31062 Toulouse, France \\ ${ }^{4}$ Laboratoire de Physique Théorique et Modélisation, CNRS UMR 8089, \\ Université de Cergy-Pontoise, F-95000 Cergy-Pontoise, France.
}

(Dated: October 27, 2018)

\begin{abstract}
We investigate the effect of the anisotropy between the s-wave scattering lengths of a threecomponent atomic Fermi gas loaded into a one-dimensional optical lattice. We find four different phases which support trionic instabilities made of bound states of three fermions. These phases distinguish themselves by the relative phases between the $2 k_{F}$ atomic density waves fluctuations of the three species. At small enough densities and strong anisotropies we give further evidences for a decoupling and the stabilization of more conventional BCS phases. Finally our results are discussed in light of a recent experiment on ${ }^{6} \mathrm{Li}$ atoms.
\end{abstract}

PACS numbers: $\quad$ 03.75.Mm, 71.10.Pm, 71.10.Fd,

Ultracold multicomponent atomic Fermi gases have recently attracted much interest [1]. In particular the existence of several internal degrees of freedom might stabilize some exotic phases. In this respect recent theoretical investigations strongly support the formation of a molecular state made of bound states of $N$ atoms. For instance quartet $(N=4)$ and trionic $(N=3)$ states have been predicted in both three and one dimensions in the context of cold atoms systems [2, 3, , 4, , 5, 6, 7, , 8, 9, 10]. However, these first studies assumed at least an $\mathrm{SU}(2)$ symmetry and even an $\mathrm{SU}(N)$ symmetry between the species which may not describe accurately the experimental situation at non-zero magnetic field. Indeed in a recent experiment, where a stable $N=3$ component mixture of atoms in three different hyperfine states of ${ }^{6} \mathrm{Li}$ has been stabilized at small magnetic field [11], the s-wave scattering lengths $a_{m n}$ between the three species exhibit strong anisotropic behavior as a function of the external magnetic field. In view of the promising perspective to observe trionic bound states in a near future, a careful study of the generic asymmetry between the species is clearly most wanted. It is the purpose of this work to do so. To this end we will study a three-component fermionic gas with equal densities, $\bar{\rho}_{1,2,3}=\bar{\rho}$, loaded into a one-dimensional (1D) optical lattice of wavelength $\lambda$ and transverse size $a_{\perp}$. Away from resonance and when the 3D scattering lengths $\left|a_{m n}\right| \ll\left(\lambda, a_{\perp}\right)$, the system is described with a Hubbard-like model with contact interactions [12]:

$$
\mathcal{H}=-t \sum_{i, n}\left[c_{i, n}^{\dagger} c_{i+1, n}+\mathrm{H} . c\right]+\sum_{i, n<m} U_{m n} \rho_{i, n} \rho_{i, m},
$$

where $c_{i, n}^{\dagger}$ is the creation operator for a fermionic atom of color $n=(1,2,3)$ at site $i$ and $\rho_{i, n}=c_{i, n}^{\dagger} c_{i, n}$ is the local density of the atomic specy $n$. The Hamiltonian (10) is an anisotropic deformation of the U(3) Hubbard model, obtained when $U_{m n}=U$, whose phase diagram has been recently elucidated [6]. In this case, for an attractive interaction $U<0$, a spectral gap opens for the $\mathrm{SU}(3)$ spin degrees of freedom and one- and two-particle excitations are gapped for incommensurate density $\bar{\rho}$. The dominant fluctuations consist into gapless Atomic Density Waves (ADW) and $\mathrm{SU}(3)$-singlet trionic excitations $\left(T_{0, i}^{\dagger}=c_{i, 1}^{\dagger} c_{i, 2}^{\dagger} c_{i, 3}^{\dagger}\right)[6]$. When $U_{12} \neq U_{23} \neq U_{31}$, the continuous symmetry of (11) is strongly reduced to $\mathrm{U}(1)^{3}$ and the resulting anisotropy has dramatic consequences. Indeed, on top of the previous symmetrical phase, we find by means of combined low-energy and density matrix renormalization group (DMRG) approaches [13, 14] that there exists for incommensurate density $\bar{\rho}$ three different ADW phases supporting trionic instabilities and even decoupled BCS phases.

The $(U, V)$ model. Let us first start with the simplest symmetry breaking pattern, $\mathrm{U}(3) \rightarrow \mathrm{U}(2) \times \mathrm{U}(1)$, when two species, say 1 and 2, play an equivalent role. In this case $U_{12}=U, U_{23}=U_{31}=V$ and (11) may be viewed as a two-component fermionic Hubbard model with coupling $U$ between the species $(1,2)$ which interacts with a third specy 3 with coupling $V$. As it will be discussed later, this model captures the essential features of the generic case. In the weak-coupling limit, its low-energy effective theory can be expressed in terms of the collective fluctuations of the densities of the three species by the bosonization approach [13]. Introducing three bosonic fields $\phi_{n}(x)$, the density operators for each specy read as follows:

$$
\rho_{i, n} \sim \frac{\bar{\rho}}{a}+\frac{\partial_{x} \phi_{n}(x)}{\sqrt{\pi}}-\frac{1}{\pi a} \sin \left[2 k_{F} x+\sqrt{4 \pi} \phi_{n}(x)\right],
$$

where $x=i a, a=\lambda / 2$ is the optical lattice spacing, and $k_{F}=2 \pi \bar{\rho} / \lambda$ is the Fermi wave-vector. The second and last terms of Eq. (2) describe respectively the uniform and $2 k_{F}$ fluctuations of the density operator of specy $n=$ $1,2,3$. In our problem the interaction is best expressed in terms of the collective fluctuations of the total den- 
sity, described by a bosonic field $\Phi_{0}=\left(\sum_{n=1}^{3} \phi_{n}\right) / \sqrt{3}$, and of the relative density, described by a two-component bosonic field $\vec{\Phi}=\left(\Phi_{\|}, \Phi_{\perp}\right)$ where $\Phi_{\|}=\left(\phi_{1}-\phi_{2}\right) / \sqrt{2}$ and $\Phi_{\perp}=\left(\phi_{1}+\phi_{2}-2 \phi_{3}\right) / \sqrt{6}$. In terms of these variables the effective low-energy Hamiltonian of the $(U, V)$ model splits into three parts, $\mathcal{H}=\mathcal{H}_{0}+\mathcal{H}_{\mathrm{s}}+\mathcal{H}_{\text {mix }}$, where:

$$
\mathcal{H}_{0}=\frac{v_{0}}{2}\left[\frac{1}{K}\left(\partial_{x} \Phi_{0}\right)^{2}+K\left(\partial_{x} \Theta_{0}\right)^{2}\right]
$$

is the Hamiltonian of a Luttinger Liquid (LL) describing the low-energy properties of the total density fluctuations. In Eq. (3), $\Theta_{0}$ is the dual field to $\Phi_{0}, v_{0}=v_{F} / K$ denotes the density velocity $\left(v_{F}=2 t a \sin \left(k_{F} a\right)\right.$ being the Fermi velocity), and $K=\left(1+2(U+2 V) a / 3 \pi v_{F}\right)^{-1 / 2}$ is the Luttinger parameter. The Hamiltonian $\mathcal{H}_{\mathrm{s}}$ accounts for the remaining (spin) degrees of freedom and reads:

$$
\begin{gathered}
\mathcal{H}_{\mathrm{s}}=\sum_{\mu=\|, \perp}\left[\frac{v_{F}}{2}\left(\left(\partial_{x} \Phi_{\mu}\right)^{2}+\left(\partial_{x} \Theta_{\mu}\right)^{2}\right)+\lambda_{\mu}\left(\partial_{x} \Phi_{\mu}\right)^{2}\right] \\
-\frac{2 g_{\perp}}{\pi a^{2}} \cos \sqrt{2 \pi} \Phi_{\|} \cos \sqrt{6 \pi} \Phi_{\perp}-\frac{g_{\|}}{\pi a^{2}} \cos \sqrt{8 \pi} \Phi_{\|},(4)
\end{gathered}
$$

with $\lambda_{\|}=g_{\|}=-U a / 2 \pi, \lambda_{\perp}=(U-4 V) a / 6 \pi$ and $g_{\perp}=-V a / 2 \pi$. Finally $\mathcal{H}_{\text {mix }}$ couples spin and density fluctuations with $\mathcal{H}_{\text {mix }}=\lambda_{\text {mix }} \partial_{x} \Phi_{0} \partial_{x} \Phi_{\perp}$ where $\lambda_{\text {mix }}=\sqrt{2}(U-V) a / 3 \pi$. When $U=V$, i.e. $\lambda_{\text {mix }}=0$, the spin and density fluctuations separate at low energy, and model (44) is the bosonized version of the $\mathrm{SU}(3)$ Gross-Neveu (GN) model studied in Ref. 6. In all other cases, $\lambda_{\text {mix }} \neq 0$, and the spin and total density degrees of freedom do not decouple, due to the anisotropy, even though we are considering incommensurate densities. However, as we will see, at weak-enough couplings, i.e. when $\left|\lambda_{\text {mix }} / 2 \pi v_{F}\right| \ll 1$, thanks to the opening of a spectral gap for the spin degrees of freedom, the spindensity coupling $\mathcal{H}_{\text {mix }}$ has little effect and can be safely neglected. In this regime the low-energy properties of the $(U, V)$ model are captured by those of $\mathcal{H}_{\mathrm{s}}$ that can be elucidated by means of a one-loop Renormalization Group (RG) approach. For generic values of the couplings $(U, V)$ we find that $\left(\lambda_{\mu}, g_{\mu}\right), \mu=(\|, \perp)$, flow to strong couplings and the three species are strongly correlated. In the strong-coupling regime, the bosonic fields $\vec{\Phi}(x)$ get locked and a spin-gap opens. We further distinguish between two phases, $\mathcal{A}_{0}$ and $\mathcal{A}_{\pi}$, depending on the sign of $V$. The $\mathcal{A}_{0}$ phase is obtained for $V<0$ and $\langle\vec{\Phi}(x)\rangle=(0,0)$ whereas the $\mathcal{A}_{\pi}$ phase is stabilized for $V>0$ with $\langle\vec{\Phi}(x)\rangle=(\sqrt{\pi / 2}, 0)$. In both phases the low-energy spectrum is an adiabatic deformation of that of the $\mathrm{SU}(3)$ GN model and consists into three kinks (and anti-kinks) $\left|\omega_{n}\right\rangle, n=(1,2,3)$ [15]. Under the $\mathrm{SU}(2)$ group acting on the species $(1,2)$, these three kinks decompose into a doublet $\left(\left|\omega_{1}\right\rangle,\left|\omega_{2}\right\rangle\right)$ and a singlet $\left|\omega_{3}\right\rangle$ with masses and velocities $\left(m_{\|}, v_{\|}\right)$and $\left(m_{\perp}, v_{\perp}\right)$ respectively. Though their wave functions are different in the two phases, they are labelled by the same quantum numbers as those of the original lattice fermions $c_{i, n}^{\dagger}$. We thus find that the one- and two-particle excitations are fully gapped in $\mathcal{A}_{0, \pi}$ phases. As a consequence the equal-time Green functions, $G_{n}(x)=\left\langle c_{i, n}^{\dagger} c_{i+x, n}\right\rangle$, are short ranged with $G_{1(2)}(x) \sim \sin \left(k_{F} x\right) \mathrm{e}^{-m_{\|} v_{\|}|x|}$, $G_{3}(x) \sim \sin \left(k_{F} x\right) \mathrm{e}^{-m_{\perp} v_{\perp}|x|}$. Furthermore defining $P_{n m}(x)=\left\langle P_{i, n m}^{\dagger} P_{i+x, n m}\right\rangle$ with $P_{i, n m}^{\dagger}=c_{i, n}^{\dagger} c_{i, m}^{\dagger}$, we find: $P_{12}(x) \sim \mathrm{e}^{-m_{\perp} v_{\perp}|x|}$ and $P_{31(2)}(x) \sim \mathrm{e}^{-m_{\|} v_{\|}|x|}$, so that neither the $\mathcal{A}_{0}$ nor the $\mathcal{A}_{\pi}$ phase support BCS pairing instabilities. The dominant fluctuations rather consist into $2 k_{F}$ ADW with correlations $N_{n m}(x)=\left\langle\rho_{i, n} \rho_{i+x, m}\right\rangle$ and trionic excitations made of three fermions.

Atomic density waves and trions. In $\mathcal{A}_{0, \pi}$ phases, upon integrating out the spin degrees of freedom, local density operators (2) simplify as:

$$
\rho_{i, n} \sim \frac{\bar{\rho}}{a}+\frac{\partial_{x} \Phi_{0}(x)}{\sqrt{3 \pi}}+\Delta_{n} \sin \left[2 k_{F} x+\sqrt{4 \pi / 3} \Phi_{0}(x)\right],
$$

where the amplitudes $\Delta_{1}=\Delta_{2}=\Delta_{\|}$and $\Delta_{3}=\Delta_{\perp}$ are non-universal functions of the couplings $(U, V)$ and are in general different. We thus find in both phases a powerlaw decay for the ADW equal-time correlations functions: $N_{n m}(x) \sim \bar{\rho}^{2}+\Delta_{n} \Delta_{m} \cos \left(2 k_{F} x\right)|x|^{-2 K / 3}$. However the two phases $\mathcal{A}_{0}$ and $\mathcal{A}_{\pi}$ distinguish themselves by the relative sign of the amplitudes $\Delta_{n}$. Indeed, we find that in the $\mathcal{A}_{0}$ phase $\Delta_{\|} \Delta_{\perp}>0$ and consequently that the $2 \mathrm{k}_{F}$ ADW of the species $(1,2)$ are in phase with that of the specy 3 . In contrast, in the $\mathcal{A}_{\pi}$ phase, we have $\Delta_{\|} \Delta_{\perp}<0$ and the $2 \mathrm{k}_{F}$ ADW of the species $(1,2)$ are out of phase from that of the specy 3 . On top of these ADWs, $\mathcal{A}_{0, \pi}$ phases support trionic excitations made of three fermions with binding energy $E_{b} \sim m_{\perp} v_{\perp}^{2}$. These excitations can also be distinguished in $\mathcal{A}_{0, \pi}$ phases but in a weaker sense. In $\mathcal{A}_{0}$ the dominant trions are characterized by the equal-time correlation function $T_{0}(x)=\left\langle T_{0, i}^{\dagger} T_{0, i+x}\right\rangle \sim$ $T_{0} \sin \left(k_{F} x\right)|x|^{-(K+9 / K) / 6}$ which is quasi-long ranged. In $\mathcal{A}_{\pi}$ the trionic wave function with maximal $k_{F}$ amplitude is obtained when two atoms $(1,2)$ at one lattice site $i$ bind antisymmetricaly with the third specy 3 at two neighborings sites $i-1$ and $i+1: T_{\pi, i}^{\dagger}=c_{i, 1}^{\dagger} c_{i, 2}^{\dagger}\left(c_{i-1,3}^{\dagger}-c_{i+1,3}^{\dagger}\right)$. Its equal-time correlation function is given by $T_{\pi}(x)=$ $\left\langle T_{\pi, i}^{\dagger} T_{\pi, i+x}\right\rangle \sim T_{\pi} \sin \left(k_{F} x\right)|x|^{-(K+9 / K) / 6}$ so that both symmetric and antisymmetric trionic correlation functions always display a power-law decay and only their amplitudes depend on phases: $\left|T_{0}\right|>\left|T_{\pi}\right|$ in $\mathcal{A}_{0}$ and $\left|T_{\pi}\right|>\left|T_{0}\right|$ in $\mathcal{A}_{\pi}$. The key quantity that distinguishes between $\mathcal{A}_{0}$ and $\mathcal{A}_{\pi}$ phases is thus the relative sign of the $2 \mathrm{k}_{F}$ amplitudes $\Delta_{\|}, \Delta_{\perp}$ of the local ADWs (5). In this respect, when going from the $\mathcal{A}_{\pi}$ to the $\mathcal{A}_{0}$ phase, a quantum phase transition (QPT) takes place on the critical line $V=0$ where $\Delta_{\|}$and $\Delta_{\perp}$ vanish and change their relative sign. There are two different QPT depending on the sign of $U$. In the type-I transition with $U>0$, all degrees of freedom become massless at the transition and 


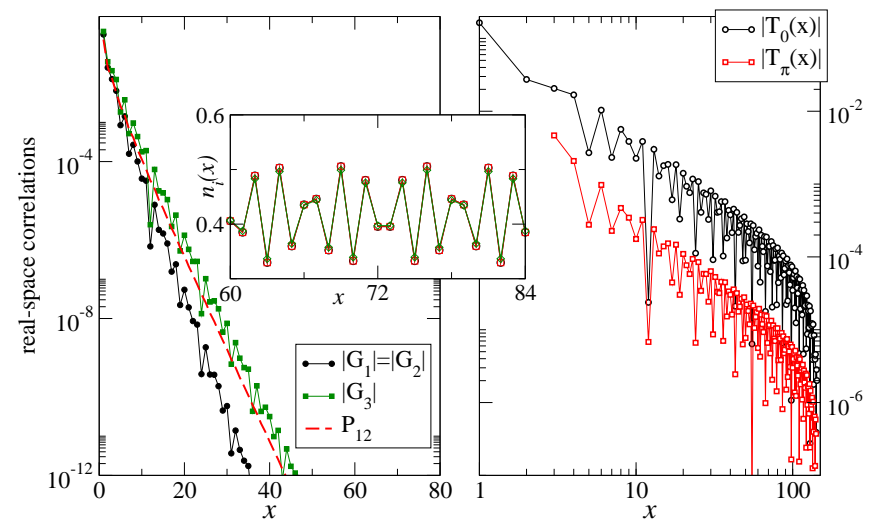

FIG. 1: (Color online) DMRG results for $(U / t, V / t)=$ $(-4,-2)$ and $\bar{\rho}=5 / 12$ in the $\mathcal{A}_{0}$ phase. Both one-particle Green functions $G_{n}$ and BCS pairing correlations $P_{12}$ are short range, while trionic correlations decay algebraically. Note that symmetric trions dominate with $\left|T_{0}\right|>\left|T_{\pi}\right|$ and local densities of all species $n_{i}(x)$ are in-phase.

the critical theory consists of three decoupled LLs. In the type-II transition for $U<0$, a QPT occurs in the twocomponent LL universality class where $m_{\|} \neq 0$ and only $m_{\perp}$ vanishes. In this case, the specy 3 decouples from the two others which form well defined BCS pairs with quasi-long range pairing correlations $P_{12}(x) \sim|x|^{-\alpha}, \alpha$ being some non-universal exponent.

Strong-couplings and Trionic-BCS transition. So far we have neglected the spin-density coupling $\mathcal{H}_{\text {mix }}$. At weak couplings, when $\left|\lambda_{\text {mix }}\right| / 2 \pi v_{F} \ll 1$, we find that the only effect of $\mathcal{H}_{\text {mix }}$ consists into a small renormalization of the low-energy parameters and do not modify qualitatively the two-phase structure discussed above. At larger couplings, when $\left|\lambda_{\text {mix }}\right| / 2 \pi v_{F} \gg 1$, the structure of the $\mathcal{H}_{\text {mix }}$ term strongly suggests that it may be responsible for a decoupling between the pair $(1,2)$ and the specy 3 leading, on top of $\mathcal{A}_{0, \pi}$ phases, to two additional phases: a BCS phase where atoms $(1,2)$ bind into pairs and even a fully gapless phase of three decoupled LLs. In the limit of large attractive $|U| / t \gg 1$ and repulsive $V / t>0$, a trionic-BCS QPT occurs from an $\mathcal{A}_{\pi}$ phase to a decoupled BCS phase in the $(1,2)$ channel at small enough densities [16]. Apart from this case, the question of how do the four phases, $\mathcal{A}_{0}, \mathcal{A}_{\pi}$, BCS and LLs, interpolate in the strong coupling or low density regime is a difficult problem which requires a thorough numerical approach like DMRG calculations.

Numerical simulations. In order to check the above theoretical predictions, we have performed extensive DMRG calculations for various densities $1 / 12 \leq \bar{\rho} \leq$ $5 / 12$ and couplings $-4 \leq U / t, V / t \leq 4$. Simulations are done on open chains (up to 144 sites) keeping up to 1600 states. The complete phase diagram will be published elsewhere [16] and we only report here our main findings. At sufficiently large densities and weak anisotropies

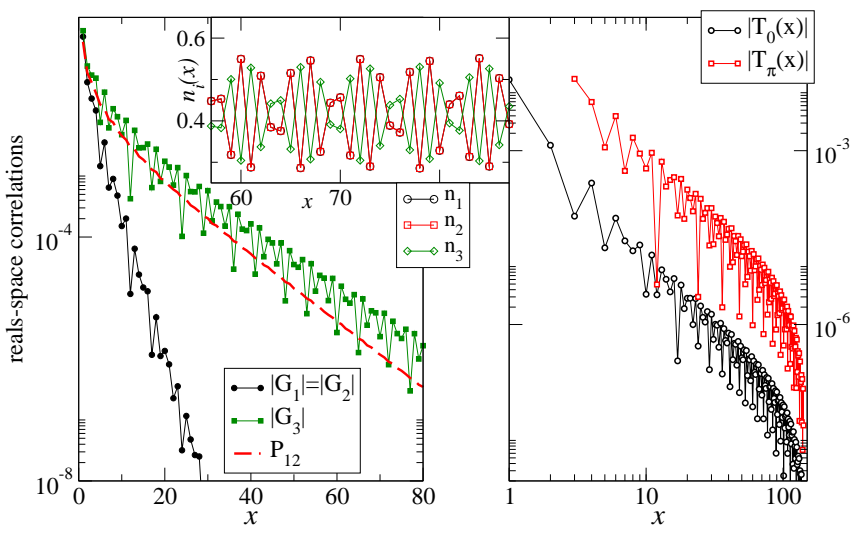

FIG. 2: (Color online) Same as Fig. 1 for $(U / t, V / t)=(-4,2)$ and $\bar{\rho}=5 / 12$ in the $\mathcal{A}_{\pi}$ phase. The only difference in that case is that antisymmetric trions dominate with $\left|T_{\pi}\right|>\left|T_{0}\right|$ and local densities $n_{1}$ and $n_{2}$ are out of phase with $n_{3}$.

the DMRG results strongly support the two phase structure, $\mathcal{A}_{0}$ and $\mathcal{A}_{\pi}$, predicted by the weak-coupling approach. As an example Fig. 1 and 2 show our results for $G_{n}(x), P_{n m}(x), T_{0, \pi}(x)$, as well as the local density profiles $n_{n}(x)=\left\langle\rho_{i, n}\right\rangle$ for a density $\bar{\rho}=5 / 12$ and typical values of the couplings in the $\mathcal{A}_{0}$ and $\mathcal{A}_{\pi}$ phases. At small densities and larger anisotropies we observe a strong tendency toward decoupling. For example, by lowering the density at fixed couplings $(U / t, V / t)=(-4,4)$, we find a QPT toward a decoupled BCS phase in the $(1,2)$ channel at densities $\bar{\rho}<\bar{\rho}_{c} \sim 1 / 4$ [17].

General asymmetric model. We are now in a position to discuss the general case where $U_{12} \neq U_{23} \neq U_{31}$. The resulting phase diagram in the parameters space is rich and complex and will be presented in details elsewhere [16]. It can be shown that at large length scales, the low-energy theory is then equivalent to that of an effective $(U, V)$ model. Since there are three inequivalent ways to define such a model, we find that, on top of the $\mathcal{A}_{0}$ phase, three inequivalent $\mathcal{A}_{\pi}(n, m)$ phases can be stabilized. The properties of each of these phases follow from those discussed above for the case $(n, m)=(1,2)$ by a suitable permutation of the indices in the correlation functions. At large couplings and/or small densities, the system decouples and three $\operatorname{BCS}(n, m)$ phases can be stabilized as well as a fully gapless decoupled LL phase.

Experimental realization. A stable mixture made of a balanced population of three hyperfine states of ${ }^{6} \mathrm{Li}$ atoms, $\left|F, m_{F}\right\rangle=|1\rangle=|1 / 2,1 / 2\rangle,|2\rangle=|1 / 2,-1 / 2\rangle$, and $|3\rangle=|3 / 2,-3 / 2\rangle$, has been stabilized recently in an optical dipole trap [11, 18]. One may in principle further load the atoms in a 3D optical lattice with potential: $V(x, y, z)=s_{\perp} E_{R}\left[\sin ^{2}(k x)+\sin ^{2}(k y)\right]+s_{\|} E_{R} \sin ^{2}(k z)$ where $s_{\perp, \|}=V_{0 \perp, \|} / E_{R}, E_{R}=\hbar^{2} k^{2} / 2 M$ being the recoil energy. A $1 \mathrm{D}$ optical lattice in the $z$ direction would then be further stabilized by increasing the lattice potential to a high enough value $s_{\perp} \gg s_{\|}$and $s_{\perp} \gg 1$. Neglecting 


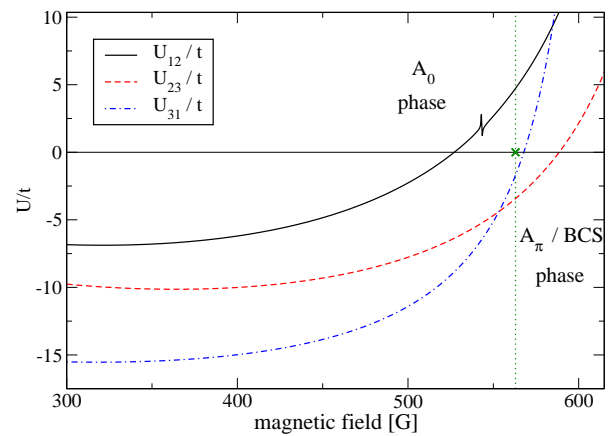

FIG. 3: (Color online) Effective Hubbard parameters $U_{n m}$ as a function of magnetic field. The cross indicates the critical field $B_{c}$ between $\mathcal{A}_{0}$ and $\mathcal{A}_{\pi}$ (or BCS) phases.

the harmonic potential and for small enough scattering lengths $a_{m n}$, the low-energy physics of such a system is captured by the fully anisotropic Hubbard model (11) 12] with parameters $U_{n m}=\sqrt{8 / \pi} E_{R}\left(s_{\perp} s_{\|}\right)^{1 / 4} a_{1 d, m n} / a_{\perp}$ and $t=4 / \sqrt{\pi} E_{R} s_{\|}^{3 / 4} e^{-2 \sqrt{s_{\|}}}$where $a_{1 d, m n}=a_{m n} /(1-$ $\left.(C / \sqrt{2})\left(a_{m n} / a_{\perp}\right)\right)$ is the effective 1D scattering length, $a_{\perp}=\lambda / 2 \pi s^{-1 / 4}$ the transverse confinement length and $C=1.4603[19]$. We show in Fig. 3 the dependence of the ratio $U_{m n} / t$ as a function of the external magnetic field $B$ for typical optical lattice parameters $\lambda=1 \mu \mathrm{m}$, $s_{\perp}=20$ and $s_{\|}=4$. Using the one-loop RG approach discussed above and large scale DMRG calculations, we find the following phase diagram which is depicted in Fig. 3. An $\mathcal{A}_{0}$ phase with symmetric trions is stabilized independently of the density for magnetic fields $B<B_{c}$. Above $B_{c}$ and at large enough densities $\bar{\rho}$ an $\mathcal{A}_{\pi}(2,3)$ phase emerges. The latter phase is unstable toward decoupling when decreasing the density below $\bar{\rho}<1 / 3$. In the decoupled phase a BCS instability occurs with pairs of atoms in states 2 and 3, the specy 1 being decoupled. The critical field is estimated with the help of RG equations to be $B_{c} \sim 563 \mathrm{G}$, a value which is consistent with our numerical data. The numerical values of the trionic binding energy strongly depend on the phases. In $\mathcal{A}_{0}$ they are mostly independent of the density and only depend on $B$. For example, we find trionic binding energies $E_{b} / k_{B} \sim 2600 \mathrm{nK}$ for $B=320 \mathrm{G}$ and $E_{b} / k_{B} \sim 100 \mathrm{nK}$ for $B=553 \mathrm{G}$ at all densities. In the $\mathcal{A}_{\pi}(2,3)$ phase (i.e. $B>B_{c}$ and $\bar{\rho}>1 / 3$ ), we find that the trionic binding energies are small (typically $E_{b} / k_{B}<30 \mathrm{nK}$ ). In the decoupled case (i.e. $\bar{\rho}=1 / 6$ and $B>B_{c}$ ), we estimate the BCS gap to be of the order 100nK. The different phases discussed above may be probed in experiments [10, 20] by measuring, with absorption imaging and via a series of magnetic field ramps, the average numbers of paired atoms $(\mathrm{nm})$ relative to the non interacting theory: $N_{n, m}=1 / L \int_{0}^{L} d x\left[\left\langle\rho_{n}(x) \rho_{m}(x)\right\rangle-\bar{\rho}^{2}\right]$. In a decoupled BCS phase with pairs in the $(n, m)$ channel and decoupled specy $p$, the number of bound pairs $(n, m)$ is macroscopic and one finds that in the limit of large sample size $L, N_{n, m} \neq 0$ whereas $N_{m, p}=N_{p, n}=0$. In both trionic phases all atoms are bound into pairs and $N_{m, n} \neq 0, N_{m, p} \neq 0$ and $N_{p, n} \neq 0$. Though in the $\mathcal{A}_{0}$ phase all $N_{n, m}$ 's are positive reflecting the presence of symmetrical trions lying on the same lattice site, in the $\mathcal{A}_{\pi}(n, m)$ phases we find $N_{n, m}>0$ but $N_{m, p}<0$ as well as $N_{p, n}<0$ reflecting the fact that the atoms of specy $p$ lie on neigboring sites where the pairs $(n, m)$ sit. In addition, there remains to discuss the effect of the three-body losses 11] which will reduce the lifetime of the trionic $A_{0}$ phase, but are expected to have little effect on the $A_{\pi}$ or BCS phases. Therefore, provided that the temperature is low enough, current available experiments could achieve a BCS pairing instability in the $(2,3)$ channel at small density or a $\mathcal{A}_{\pi}(2,3)$ phase for larger densities.

We thank T. Ottenstein et al. for sharing their experimental data. Discussions with E. Boulat, V. Dubois, G. Roux, C. Salomon, G.V. Shlyapnikov, A.M. Tsvelik, and S.R. White are also aknowledged. S.C. thanks CALMIP (Toulouse) and IDRIS (Paris) for allocation of cpu time.

[1] A. G. K. Modawi and A. J. Leggett, J. Low Temp. Phys. 109, 625 (1997); R. W. Cherng, G. Refael, and E. Demler, Phys. Rev. Lett. 99, 130406 (2007).

[2] P. Lecheminant, E. Boulat, and P. Azaria, Phys. Rev. Lett. 95, 240402 (2005).

[3] C. J. Wu, Phys. Rev. Lett. 95, 266404 (2005).

[4] H. Kamei and K. Miyake, J. Phys. Soc. Jpn. 74, 1911 (2005).

[5] A. Rapp et al., Phys. Rev. Lett. 98, 160405 (2007); A. Rapp, W. Hofstetter, and G. Zaránd, Phys. Rev. B 77, 144520 (2008).

[6] S. Capponi et al., Phys. Rev. A 77, 013624 (2008).

[7] X.-J. Liu, H. Hu, and P. D. Drummond, Phys. Rev. A 77, 013622 (2008).

[8] X. W. Guan et al., Phys. Rev. Lett. 100, 200401 (2008).

[9] G. Roux et al., Eur. Phys. J. B 68, 293 (2009).

[10] R. A. Molina, J. Dukelsky, and P. Schmitteckert, Phys. Rev. A 80, 013616 (2009).

[11] T. B. Ottenstein et al., Phys. Rev. Lett. 101, 203202 (2008).

[12] D. Jaksch and P. Zoller, Ann. Phys. (N.Y.) 315, 52 (2005).

[13] A. O. Gogolin, A. A. Nersesyan, and A. M. Tsvelik, Bosonization and Strongly Correlated Systems (Cambridge University Press, Cambridge, England, 1998).

[14] S. R. White, Phys. Rev. Lett. 69, 2863 (1992).

[15] N. Andrei and J. H. Lowenstein, Phys. Lett. B 90, 106 (1980).

[16] P. Azaria et al., in preparation.

[17] See EPAPS Document No. for supplementary data. For more information on EPAPS, see http://www.aip.org/pubservs/epaps.html

[18] J. H. Huckans et al., Phys. Rev. Lett. 102, 165302 (2009).

[19] M. Olshanii, Phys. Rev. Lett. 81, 938 (1998).

[20] R. Jördens et al., Nature 455, 204 (2008). 